\title{
Growth Rate Exponents of Richtmyer-Meshkov Mixing Layers
}

Y. Zhou and T. Clark

This article was presented to:

Physical Review

Lawrence

Livermore

National

Laboratory

January 2004 


\section{DISCLAIMER}

This document was prepared as an account of work sponsored by an agency of the United States Government. Neither the United States Government nor the University of California nor any of their employees, makes any warranty, express or implied, or assumes any legal liability or responsibility for the accuracy, completeness, or usefulness of any information, apparatus, product, or process disclosed, or represents that its use would not infringe privately owned rights. Reference herein to any specific commercial product, process, or service by trade name, trademark, manufacturer, or otherwise, does not necessarily constitute or imply its endorsement, recommendation, or favoring by the United States Government or the University of California. The views and opinions of authors expressed herein do not necessarily state or reflect those of the United States Government or the University of California, and shall not be used for advertising or product endorsement purposes.

This is a preprint of a paper intended for publication in a journal or proceedings. Since changes may be made before publication, this preprint is made available with the understanding that it will not be cited or reproduced without the permission of the author. 


\title{
Growth rate exponents of Richtmyer-Meshkov mixing layers
}

\author{
Timothy T. Clark \\ Los Alamos National Laboratory \\ Los Alamos, New Mexico 87545. \\ Ye Zhou \\ Lawrence Livermore National Laboratory \\ Livermore, California 94551.
}

\begin{abstract}
The Richtmyer-Meshkov mixing layer is initiated by the passing of a shock over an interface between fluid of differing densities. The energy deposited during the shock passage undergoes a relaxation process during which the fluctuational energy in the flow field decays and the spatial gradients of the flow field decrease in time. This late stage of Richtmyer-Meshkov mixing layers is studied from the viewpoint of self-similarity. Analogies with weakly anisotropic turbulence suggest that both the bubble-side and spike-side widths of the mixing layer should evolve as power-laws in time, with the same power-law exponents and virtual time origin for both sides. The analogy also bounds the power-law exponent between $2 / 7$ and $2 / 5$. It is then shown that the assumption of identical power-law exponents for bubbles and spikes yields that are in good agreement with experiment at modest density ratios.
\end{abstract}

PACS numbers: 47.27. Eq, 47.27. Gs, $47.40 \mathrm{Nm}$, 52.57.Fg 


\section{INTRODUCTION}

The Richtmyer-Meshkov (RM) instability occurs when a perturbed interface between two fluids of differing densities is impulsively accelerated. The impulsive acceleration is typically produced by a shock wave passing across the interface, but may be induced by other means. The RM instability is of significant importance in problems of inertial confinement fusion as well as in stellar evolution. The RM instability was studied analytically by Richtmyer[1] and experimentally by Meshkov [2]. The early-time behavior is amenable to linear analysis[1, 3]. The evolution in the case of a single Fourier-mode perturbation is also relatively well understood. However, during the late stages of the evolution of the RM mixing layer initiated from a multi-modal initial condition, the mixing layer may become highly turbulent, during which time a statistical description becomes more appropriate. The typical statistical characterization is of the width of the mixing layer as a function of time, and more specifically the "bubble" (light fluid penetrating heavier fluid) and "spike" (heavy fluid penetrating lighter fluid) growth rates.

The usual scaling assumptions regarding the bubble- and spike-growth rates are based on empirical observation. These assumptions are that the bubble-side and spike-side widths $\left(h_{B}\right.$ and $h_{S}$, respectively) grow as power-laws in time, with the bubble-side having a somewhat smaller exponent, $\theta_{B}$ than the spike-side $\theta_{S}$;

$$
h_{B, S}(t) \approx t^{\theta_{B, S}}, \theta_{B}<\theta_{S} .
$$

Recently, Zhou[4] has presented an analysis that draws a connection between the exponents and the growth of the length-scale in a decaying, weakly anisotropic turbulence. The physical picture presented by Zhou is one of an initially quiescent flow field subject to an impulsive injection of energy (perhaps at all scales) and then subsequently allowed to freely decay. Although there are undoubtedly significant corrections due to strong anisotropy and lack of an equilibration of the statistical quantities at early time, the analogy between the RM induced flow and decaying isotropic turbulence becomes better and better as the shockinduced anisotropy decays toward isotropy after the shock passes. As a result, one can anticipate that the analogy between isotropic decay and RM mixing layer evolution will be acceptable at a late time (long after the shock passage). Indeed, it is at late time that one expects that mixing zone width of the RM induced flow will grow as a power-law in time. The fact that the mixing zone width is the largest observable feature of the flow suggest the 
importance of a dominant large length scale, which, in turn, is associated with the low-wave number power-law of the energy spectrum. Such a viewpoint is very similar to the analytical and experimental findings in isotropic turbulence beginning with Batchelor [5], Saffman[6], and productively exploited by others, e.g., Comte-Bellot and Corrsin[7].

\section{SELF-SIMILAR SCALING ANALYSIS}

The late-time evolution of the RM mixing layer will be studied using the analogy with isotropic turbulence to generate a a new and different view. This analogy indicates that both the bubble- and spike- side widths of the mixing layer should evolve as power-laws in time, with the same power-law exponent for both sides. This is a direct outcome from turbulence theory which implies that the correlation length between fluid in the spikes and bubble at large separation distances should be the same. Therefore, our result provides an insight that may explain the observed late-time behaviors of these layers and suggests that the asymmetry of the motion of bubbles and spikes should be instead attributed to the virtual time origins and coefficients for each side. In the following sections the self-similar decay of isotropic turbulence will be reviewed, the qualitative features of the analogy to RM decay will be developed, and the consequences for mixing layer growth rates will be determined.

\section{A. Review of self-similarity of isotropic turbulence}

A review of the nature and mathematical basis of self-similarity for isotropic turbulence will be reviewed with the goal of establishing the necessary scaling arguments and introducing the concepts used in the later analysis.

The decay of isotropic turbulence has been extensively studied over the past 70 years, beginning with the seminal work of Kármán and Howarth[8] in 1938. Various theoretical descriptions, dimensional analyses, computational simulations and experiments have been conducted over the years. The physical picture that has arisen is described in detail by Lesieur[9] and Lesieur and Schertzer[10]. Initially random isotropic velocity fluctuations

rapidly "build-up" an inertial subrange. The inertial subrange extends to increasingly large wavenumbers as time goes on until eventually the viscous processes at high wavenumbers 
begin to destroy the energy at the high wavenumbers. Prior to the point in time when the inertial subrange extends to the viscously damped wavenumbers, the total turbulent kinetic energy is nearly conserved. After establishment of the inertial subrange the turbulence tends towards the high-Reynolds number isotropic decay regime. At very late times, the effects of viscosity will eventually affect the larger scales of motion leading to Batchelor's [5] socalled final stage of decay in which the dynamical interactions in the turbulent flow become dominated by the viscous damping.

Such an evolutionary picture of the isotropic decay was studied by Clark and Zemach using spectral closure models and a simple group-theoretic approach [11, 12]. Their approach was based on a mathematical definition of self-similarity-self-similar solutions are those that are invariant under an appropriate symmetry group. For the case of decaying isotropic turbulence turbulence the appropriate scaling group consisted of a simple time-scaling with time-translation;

$$
t^{\prime}=\tau\left(t+t_{0}\right)-t_{0}
$$

and to a simple scaling of length;

$$
\mathcal{L}^{\prime}=\lambda \mathcal{L}
$$

The existence of a virtual time origin, $t_{0}$ is a statement of time-translation invariance-the behavior of the system should be independent of the absolute time of the "clock" used to measure the system. It will be shown later that $t_{0}=0$ is an admissible solution. Clark and Zemach found that the same group was also appropriate for decaying weakly anisotropic turbulence-the fundamental scaling properties of the deviatoric parts of the spectrum being identical to those of the trace.

This two-parameter group was restricted to a simple power-law subgroup;

$$
\lambda=\tau^{\theta}
$$

This subgroup $(\tau, \theta)$ implies that changes in lengths are related changes in time by a power law. Using this subgroup in addition to the above scaling, the generally accepted power-law behaviors for decaying isotropic turbulence were derived. The simple group-theoretic argument also showed that the return to isotropy of an initially anisotropic flow was incomplete-a residual amount of anistropy persists at all times. This conclusion was also supported by computations with a spectral closure model[11]. 
Clark and Zemach [12] exploited the subgroup given by eqn. 2.3 to determine the form of the self-similar energy spectrum,

$$
E(k, t)=K(t) L(t) f(k L(t))
$$

where $K(t)$ is the turbulent kinetic energy, $L(t)$ is a characteristic length-scale of the energy containing range of the turbulence and $k$ is the wavenumber. This form is identical to the form postulated by de Kàrman and Howarth [8]. The analysis also yielded the self-similar form of the length-scale which was found to be

$$
L(t)=L_{0}\left[\frac{t+t_{0}}{\left|t_{0}\right|}\right]^{\theta},
$$

and the form for the turbulent kinetic energy was found to be

$$
K(t)=K_{0}\left[\frac{t+t_{0}}{\left|t_{0}\right|}\right]^{-n},
$$

where

$$
n=2-2 \theta \text {. }
$$

Self-similarity of a physical law implies not only that the scaled functions of the unscaled arguments are equal to the unscaled functions of the scaled arguments, but also that physical parameters (e.g., viscosity, acceleration et cetera) scale onto themselves, that is, that the relevant dimensional parameters remain invariant. Thus the parameter $\theta$ may be determined by requiring the physical parameters of the problem be invariant. For example, for the RT mixing layer, Clark and Zhou [13] required the acceleration, g (dimensions $\left[\right.$ Length/Time $\left.{ }^{2}\right]$ ) be made invariant;

$$
g^{\prime}=g \frac{\lambda}{\tau^{2}}=g \tau^{\theta-2}
$$

From this we see that $\theta-2=0$ or $\theta=2$ makes $g$ invariant and gives a length-scale (for example the mixing layer width) that grows as $t^{2}$. For the KH mixing layer the operative physical parameter is the velocity difference, $U_{\Delta}$;

$$
U_{\Delta}^{\prime}=U_{\Delta} \frac{\lambda}{\tau}=g \tau^{\theta-1}
$$

and $U_{\Delta}$ is invariant for $\theta=1$, giving a mixing layer width that grows as $t^{1}$.

For isotropic turbulence, Clark and Zemach considered two physical effects-viscosity and the observed persistent large-scale eddies. The invariance of viscosity requires $\theta=1 / 2$ 
leading to an energy decay law of $t^{-1}$ and length-scale growth of $t^{-1 / 2}$. The consequences of this scaling group on the "permanence of large eddies" requires particular values of the time-exponents of the functions (related to the parameter $\theta$ ). Permanence of large-eddies (or equivalently, invariance of the infrared spectrum of the energy) establishes a connection between $\theta$ and the low-wavenumber power-law behavior of the spectrum. For permanent large eddies one has,

$$
\lim _{k \rightarrow 0} E(k, t)=e_{0} k^{m},
$$

where $e_{0}$ has dimensions of $\left[\right.$ Length $h^{3+m} /$ Time $\left.^{2}\right]$ and invariance of $e_{0}$ requires that

$$
\tau^{(3+m) \theta-2} \epsilon_{0}=\epsilon_{0}
$$

which has a solution for $\theta$ of

$$
\theta=\frac{2}{m+3}
$$

and

$$
n=2 \frac{m+1}{m+3}
$$

Note that full self-similarity with viscosity $(\theta=1 / 2)$ and including permanence of large eddies, eqn. 2.12, restricts one to the choice of $m=1$, consistent with the decay laws for both the large and small scales. Speziale and Bernard [17] studied the Kármán-Howarth equation in an effort to deduce the fully self-similar form of decaying isotropic turbulence with viscosity. They concluded that for this case that the energy decay exponent was $n=1$. Ristorcelli has shown that this is consistent with constant skewness and palinstrophy in the dissipation equation [27]. Clark and Zemach[12] concurred that such a solution, if it exists, is fully self-similar and point out that this corresponds to a low wavenumber exponent of $m=1$. A value of $m<2$ corresponds to a modal energy spectrum that is singular at the origin. For isotropic, non-helical turbulence, the modal spectral tensor becomes

$$
\begin{aligned}
\lim _{k \rightarrow 0} E_{i j}(\mathrm{k}, t) & =\lim _{k \rightarrow 0} \frac{E(k, t)}{4 \pi k^{2}}\left(\delta_{i j}-\frac{k_{i} k_{j}}{k^{2}}\right) \\
& =\frac{E_{o}}{4 \pi k_{o}^{n}} k^{m-2}\left(\delta_{i j}-\frac{k_{i} k_{j}}{k^{2}}\right) .
\end{aligned}
$$

Mathematically this corresponds to an ensemble of realizations wherein some of the realizations have an infinite translational velocity, but where the average velocity for the ensemble 
is zero[20]. This does not seem physically reasonable, and we will only consider the cases of $m \geq 2$.

The low-wavenumber behavior of a turbulent flow is related to the evolution of the largest scales in the flow field (i.e., velocity correlations at large physical distances). An early work dealing with the low-wavenumber behavior of turbulence is by Batchelor [5], in which a prediction for the decay rate for isotropic turbulence was made. He assumed (implicitly) that $m=4$ for the isotropic decay. This circumstance corresponds to constancy of the Loitsianski integral [5, 9, 18] and "permanence of big eddies." As pointed out by Lesieur and Schertzer, the case of $m>4$ is modified by backscatter to $m=4$. The case of $m=4$ corresponds to a marginal self-similarity with the kinetic energy decay exponent $n \approx 1.38$ rather than $n=10 / 7$. Lesieur([9], page 194$)$ states that for $m<4$, the initial low-wavenumber spectrum of unforced turbulence remains the same. From the perspective of our paper, this implies that the turbulent growth will depend upon the initial conditions and there is one correct value for $\theta$ that is determined by the infrared scaling of the initial conditions. It must be noted that recently Eyink and Thomson[19] used the eddy-damped quasi-normal Markovian (EDQNM) model to conclude that there is a breakdown of absolute self-similarity for $m>\approx 3.45$, suggesting a modification of relationship between $m$ and $n$ and $\theta$. Corrections for backscatter where discussed by Clark and Zemach [12]. However, the difference between the fully self-similar decay-rates and the decay-rates modified by backscatter is small and need not be discussed further. We will restrict our attention to $m \leq 4$

Saffman[6] showed that for a homogeneous field generated by an impulsive force, $m=2$ and that the large-scale structure is an invariant of the flow field (i.e., the "permanence of big eddies"). As pointed out by Saffman, the case of $m=2$ corresponds to an equipartition of energy at the large-scales, implying that the turbulence (i.e., some realizations in the ensemble of realizations) must possess a net momentum.

The role of the virtual time-origin, $t_{0}$ and it's determination from experimental data were studied by Mohamed and LaRue [16]. Calculations using spectral models indicate that the initial phase of isotropic turbulence involves the establishment of a cascade, and until this is well-established the turbulence is not self-similar. Thus the theories and models demonstrate that $t_{0}$ is related to the time required to establish self-similarity after the initial excitation. 


\section{B. Self-Similarity and Richtmyer-Meshkov induced flow}

In this section the consequences of self-similarity for RM mixing layers will be determined using a simplified Lie-group analysis similar to the apporach used by Clark and Zemach [12]. After the form of the self-similarity is determined, an anology with isotropic decay will be assumed to relate the one-parameter subgroup to the infrared scaling of the mixing layer. While it is not generally believed that RM layers develop into self-similar mixing layers (particularly at large density ratios), one may still usefully explore the concept of self-similarity to attempt to deduce gross scaling behaviors.

The physical description of the RM mixing layer recently proposed by Zhou [4] is remarkably reminiscent of this view of isotropic turbulence with permanent large-scale structures, with the early deposition of energy being due to the initial impulse which drives the mixing layer. After a finite period of time, the flow relaxes to a anisotropic, inhomogeneous state. For this study we will consider late times, long after the shock has passed and we will presume that the flow field is self-similar. Implicit in this assumption is that the persistent anisotropy of the system is weak, consistent with the assumptions of Clark and Zemach [11].

Consider the self-similar growth of the width $W(t)$ of a Richtmyer-Meshkov mixing layer. The same scalings and subgroup (eq. 2.1-2.3) employed by Clark and Zemach [12] and Clark

and Zhou [13]. $W(t)$ has dimensions of length and it's single argument has dimensions of time. Self-similarity requires that the scaled function $W$ is equal to the unscaled function of the scaled argumet, $t$;

$$
\left.\lambda W(t)=W\left(\tau\left(t+t_{0}\right)-t_{0}\right)\right) .
$$

The power-law subgroup, eqn. 2.3, yields

$$
\left.\tau^{\theta} W(t)=W\left(\tau\left(t+t_{0}\right)-t_{0}\right)\right)
$$

It is generally true that eqn. 2.16 is implied for all group elements if it holds for the infinitesimal element, $1+\delta \tau$, where $\delta \tau$ is infinitesimal. Thus we may differentiate eqn. 2.16 with respect to $\tau$ and set $\tau$ to unity to yield the so-called determining equation,

$$
\theta W(t)=\left(t+t_{0}\right) \frac{d W(t)}{d t}
$$

Solving gives

$$
W(t)=W_{0}\left[\frac{t+t_{0}}{t_{0}}\right]^{\theta} .
$$


This result is analogous to that found for the length-scale of isotropic turbulence by Clark and Zemach. Of course such a form is equivalent to

$$
W(t)=\frac{W_{0}}{t_{0}^{\theta}}\left[t+t_{0}\right]^{\theta},
$$

and in the limit of $t_{0} \rightarrow 0$ we require that $W_{0} \rightarrow 0$ so that the product $W_{0} t_{0}^{-\theta}$ is a nonzero constant. This limiting case is the form which is generally used to analyze RM experimental data.

Applying this approach to the energy spectrum, the turbulent kinetic energy, and Reynolds stresses yields results that are identical to those found by Clark and Zhou [13] for the case of the RT mixing layer (up to, but not including the determination of $\theta$ ). For a mixing layer that is statistically axisymmetric in $(y, z)$ and inhomogeneous in $x$, the self-similar energy spectrum can be represented by

$$
E\left(x, k_{\perp}, t\right)=K(t) W(t) F(\chi, \xi)
$$

where $K(t)$ is given by eq. 2.6, W(t) is given by eq. 2.18. The similarity variables $\chi$ and $\xi$ are

$$
\chi=\frac{x}{W(t)}
$$

and

$$
\xi=k_{\perp} W(t)
$$

The turbulent kinetic energy at $x$ in the mixing layer is given by

$$
\mathcal{K}(x, t)=\int_{0}^{\infty} E\left(x, k_{\perp}, t\right) d k_{\perp}=K(t) \int_{0}^{\infty} F(\chi, \xi) d \xi=K(t) f(\chi)
$$

For the case of anisotropic turbulence, the self-similar spectral tensor possesses the same dimensionality as the energy tensor and consequently has an analogous form;

$$
E_{i j}\left(x, k_{\perp}, t\right)=K(t) W(t) f_{i j}(\chi, \xi)
$$

This form permits variation in the anisotropy across the layer. Note that other possible forms are not suggested by the analysis (see Clark and Zhou [13]). The Reynolds stress tensor is therefore

$$
R_{i j}(x, t)=2 \int_{0}^{\infty} E_{i j}\left(x, k_{\perp}, t\right) d \xi=K(t) \int_{0}^{\infty} F_{i j}(\chi, \xi) d \xi=K(t) F_{i j}(\chi)
$$


which implies that for the self-similar inhomogeneous anisotropic mixing layer the anistropy magnitude becomes independent of time. The anisotropy is a function of the similarity variable, $\chi$ alone,

$$
b_{i j}(x, t)=\frac{R_{i j}(x, t)-\frac{1}{3} \delta_{i j} R_{n n}(x, t)}{R_{n n}(x, t)}=\frac{F_{i j}(\chi)-\frac{1}{3} \delta_{i j} F_{n n}(\chi)}{F_{n n}(\chi)}=B_{i j}(\chi) .
$$

The self-similar decay of the energy and growth of the RM mixing layer requires the deviatoric parts of the Reynolds stress to decay at the same rate as the energy. This is also consistent with the findings of Clark and Zemach [12] for homogeneous turbulence.

The problem remains to find $\theta$. In other words, is there a physical effect or parameter governing the subsequent evolution of the layer, of which the invariance establishes the overall dynamical evolution? For the RT mixing layer the physical constraint was the acceleration. For isotropic decay, it is either the viscosity, or the permanence of the large scales. For the present circumstance, requiring invariance of the viscosity yields $\theta=1 / 2$, giving a mixing layer that grows as the square-root of time like the simple diffusion layer. The alternative is to ask what features of the flow field may be invariant during the evolution. Zhou's conjecture is consistent, at late times, with a limited form of the permanence of large eddies at the center of the mixing layer;

$$
\lim _{k \rightarrow 0} E\left(x, k_{\perp}, t\right)=\lim _{k \rightarrow 0} K(t) W(t) F(\chi, \xi)=K(t) W(t) F_{0}(\chi) \xi^{m}
$$

This is a conjecture and assumes that the function $F(\chi, \xi)$ is separable, at least for small values of $\xi$. Using eqn.s 2.18,2.22 and 2.6 this becomes

$$
K(t) W(t) F_{0}(\chi) \xi^{m}=K(t) W(t) F_{0}(\chi) k^{m} W^{m}(t)=K_{0} W_{0}^{1+m} F_{0}(\chi)\left[\frac{t+t_{0}}{t_{0}}\right]^{(3+n) \theta-2} .
$$

For the choice of $(3+n) \theta-2=0$ (which is identical to the conclusion found by Clark and Zemach for decaying isotropic turbulence, eq. 2.12) we have

$$
\lim _{k \rightarrow 0} E\left(x, k_{\perp}, t\right)=K_{0} W_{0}^{1+m} F_{0}(\chi)
$$

which is constant in time. This is the circumstance alluded to by Zhou [4]. Zhou pointed out that the admissible values for $2<m \leq 4$ give values of $\theta$ that are in approximate agreement with experimental estimations of the exponents governing the growth of the bubbles and spikes of the RM mixing layer. 


\section{Comparison of Richtmyer-Meshkov Growth rates and Decay Exponents}

The above analysis produced a charaterization of the growth rate of an RM mixing layer with two parameters-a time-exponent $\theta$ and a virtual time-origin $t_{0}$. The usual characterization for RM mixing layers is to have a virtual time-origin of $t_{0}=0$ and two different time-exponents. In this section we will first address the relationship of the above-predicted $\theta$ and the two exponents reported in most experimental studies of RTM mixing. Secondly, we will address the role of the virtual time-origin.

There exists a consensus in the community that the bubbles and spikes of the RM mixing layer follow growth rates with different exponents as shown in eqn. 1.1. The present ansatz is that the bubble and spike heights sum to give the width,

$$
h_{S}(t)+h_{B}(t)=W(t),
$$

implying that both sides should possess the same power-law behavior

$$
h_{B}(t)=h_{B 0}\left[\frac{t+t_{0}}{t_{0}}\right]^{\theta}, h_{S}(t)=h_{S 0}\left[\frac{t+t_{0}}{t_{0}}\right]^{\theta} .
$$

Self-similarity implies the correlations between fluid in the spikes at large separation distances perpendicular to the inhomogeneous direction should scale with distance the same as the correlations between bubbles at these distances. In other words, the same operative forces (pressure, acceleration et cetera) affect the heavy fluid in the same manner as they affect the light fluid. To do otherwise would require the fluctuations within the spikes be correlated in a fashion that is independent of the lighter fluid that separates them. This seems physically unreasonable at modest Atwood numbers, but indeed may be correct at

large Atwood numbers. However, if the spike and bubble correlations do scale similarly at large separation distances, it implies that the bubble- and spike-growth should have the same temporal power-law behavior. However, as stated above, empirical data are consistent with the assumption that the power-law exponents are different.

The second issue is the existence of the virtual time origin, $t_{0}$ in the present theory. The parameter $t_{0}$ arises due to the generality of the time-scaling. Had we not required timetranslation, we would not have produced solutions with the virtual origin. However, this seems overly restrictive for a general theory. Note that if one makes the arbitrary assertion that $t_{0}=0$ then the present theory requires that $W_{0}=0$ so that the ratio $W_{0} / t_{0}^{\theta}=\tilde{W}_{0}$ is 
constant. The existence of the virtual time origin is a consequence of the general theory but makes the representation look unduly flexible. One may quite easily match experimental data for various values of $\theta$ if $t_{0}$ is allowed to vary. This issue was addressed by both ComteBellot and Corrsin [7] and by Mohamed and LaRue [16] for the case of decaying isotropic turbulence. The unfortunate conclusion is that it is dauntingly difficult to simultaneously discern both the exponent and the virtual origin from either experiment or direct numerical simulations. However, this difficulty does not necessariy warrant that $t_{0}=0$.

Following Zhou[4], assume that the dominant large-scale mixing length is proportional to the turbulent length-scale. Specifically, both $h_{B}(t)$ and $h_{S}(t)$ scale in the same fashion as the length-scale $L(t)$ commonly discussed in the context of isotropic turbulence. Thus we write for the bubble-side

$$
h_{B}(t)=h_{B 0}\left[\frac{t+t_{B}}{\left|t_{B}\right|}\right]^{\theta},
$$

and for the spike side,

$$
h_{S}(t)=h_{S 0}\left[\frac{t+t_{S}}{\left|t_{S}\right|}\right]^{\theta},
$$

where $W_{0}=h_{B 0}+h_{B 0}$ and the exponent $\theta$ is related to $n$ and hence $m$, by eqn.s 2.7 and 2.12, yielding

$$
\theta=\left(1-\frac{n}{2}\right)=\frac{2}{m+3}
$$

The coefficients $h_{B 0}$ and $h_{S 0}$ are related to the scaling for the turbulence length scale by dimensional considerations and by eqn. 2.34;

$$
h_{B 0} \propto h_{S 0} \propto \frac{K_{0}^{1 / 2} t_{0}}{n}\left(=\frac{K_{0}^{1 / 2} t_{0}}{2(1-\theta)}\right) .
$$

Here the coefficients, $h_{B 0}$ and $h_{S 0}$ set the amplitude of the mixing-layer scaling laws and are unknown constants which may depend on Atwood number. The bubble and spike widths, $h_{B}(t)$ and $h_{S}(t)$ grow in a fashion assumed to be analogous to $L(t)$ and the $\lambda_{B, S}$ The virtual time origin, $t_{0}$ arising from time-translation invariance may be considered to be related to the time required for the physical processes to equilibrate toward self-similarity. Such physical effects may include the influence of Atwood numbers as well as the time required to achieve a full spectrum of turbulent fluctuations in both fluids. Our assumption that $2<m \leq 4$ implies that

$$
\frac{2}{7}<\theta \leq \frac{2}{5}
$$


It is instructive to compare these bounds to reported values of $\theta_{B}$. Alon, Hecht, Ofer, and Shvarts [21] reported a value of $\theta_{B}=0.4$, implying $m=2$, as indicated by Zhou [4]. This result is consistent with Saffman's picture of large-scale energy deposition due to impulsive forces. The equipartition of energy at largest scales (implied by Saffman's $k^{2}$ infrared energy spectra) and the so-called "permanence of big eddies" may also be a clue as to why the evolution of the RM mixing layer appears to be strongly dependent on initial conditions. More recently, Dimonte and Schneider [22, 24] and Schneider, Dimonte and Remington [23] have reported values of $\theta_{B}=0.25$ based on analysis of their experimental results. Likewise, Oron, Arazi, Rikanati, Alon and Shvarts have reported $\theta_{B}=0.25$ based on analysis and simulation. Although, this value is slightly lower than our lower bound being $\theta_{B} \approx 0.29$, it is close enough to be encouraging.

\section{NUMERICAL TESTS}

In this section we will apply the presumed self-similar forms to analyse an experiment of RM mixing layers. The analyses will first study the role of the virtual time-origin by determining the virtual origin that best fits the experimental data for a sequence of values of $\theta$. This will be performed for fits to spikes and bubbles independently, and for the total width of the mixing layer. The analyses will then study the effects of applying a single time exponent to both sides of the layer.

Finding both the correct exponent, $\theta$ and the correct virtual time origin, $t_{0}$ is a difficult task. Our approach is as follows:

1. We discretize the $\theta$-interval bounded by $(2 / 7,2 / 5)$.

2. For each discrete value of $\theta$ on the interval, we perform least-mean-square fits to the experimental data to determine the virtual origins $\left(W_{0}, t_{0}\right)$ for a fit to the width and $\left(h_{B 0}, t_{0}\right)$ and $\left(h_{S 0}, t_{0}\right)$ for fits to bubbles and spikes.

3. We use the virtual origin data to determine the goodness of fit by computing the L2-error of the fit for each of the three fits.

In order to fit negative values of the virtual time origin, we will use the formulation;

$$
\left.W^{(} t\right)=W_{0}\left[\frac{t+t_{0}}{\left|t_{0}\right|}\right]^{\theta} .
$$


We will fit the logarithm of this equations,e.g,,

$$
W^{\frac{1}{\theta}}(t)=A_{1}\left(t+t_{0}\right)=A_{1} t+A_{0}
$$

where

$$
A_{1}=\frac{W_{0}^{\frac{1}{\theta}}}{\left|t_{0}\right|}
$$

and

$$
A_{0}=W_{0}^{\frac{1}{\theta}} \frac{t_{0}}{\left|t_{0}\right|}=A_{1} t_{0}
$$

The least-mean-square fit are then made between the experimental data and the above form. Analogous forms are used to fit the bubbles and spikes independently.

We will examine the experiment of Dimonte and Schneider[24] of impulsively-driven Rayleigh-Taylor mixing layers. The set of experiments to be examined where conducted at two different Atwood numbers $\left(\rho_{2}-\rho_{1}\right) /\left(\rho_{2}+\rho_{1}\right), 0.22$ (Experimental Set $\mathrm{A}$ ) and 0.48 (Experimental Set B). Each set consisted of two independent shots. To augment the data in the fits we constrain simultaneously to the shots within each Atwood number. This is equivalent to treating the two independent shots as an ensemble, and applying the data-fit to the ensemble.

Figures 1 and 2 show the values of $t_{0}$ and the $L 2$ error of the fits of mixing layer width, bubble height and spike height versus $\theta$ for Experimental Set $A$ and B, respectively. These figures indicate that the circumstance of $t_{0}=0$ (or equivalently, $A_{0}=0$ ) do not coincide with a minimum in the error of the fit. For Experimental Set A, shown in Figure 1, the virtual time origin for the fit to the spike statistics never crosses zero for any choice of $\theta$ on the interval, and the bubble and width are zero for different values of $\theta$. In Experimental Set B, Figure 2 the fits to width, bubble and spike result in three different virtual origins. This suggests that the choice $t_{0}=0$ is not warranted from this experimental data. The trend appears to be that the smallest values of $\theta$ seem to produce the least error in the fits. This is in concordance with the values of $\theta$ reported by Alon et al. [21], Dimonte and Schneider [22, 24] and Schneider et al. [23].

Figure 3 displays the bubble and spike results for the Experimental Data Set A $(A t=$ 0.22 ) and fits to the data based on minimizing the $L 2$ error for fitting the bubble data, fitting the spike data and fitting the total width data. The results for the fit to the spike data gives the best apparent agreement at early time, but the late time results for all three constraints 
look satisfactory. Figure 4 displays the fits to the width based on data from fits for the bubbles, for the spikes and for the total width. Again, the early time data is best matched by using the spike to determine the fit parameters (yielding $\theta=0.293 \mathrm{and}_{0}=-11.974$ ), but at late times all three constraints appear adequate.

Figures 5 and 6 display the results of the fits for Experimental Set B $(A t=0.48)$. For the higher Atwood number the best agreement at small times comes from the fits based on the total width data $\left(\theta=0.286, t_{0}=-8.569\right)$. Note that the late time agreement for all three fits is again satsifactory.

Since the total width incorporates both the data for the spike-side and the bubble-side, the fits based on the width data may be considered more "robust." In addition, the best fits based on the width data yielded the same exponents for both data sets, $\theta=2 / 7$ which also coincides most closely with results of Alon et al. [21], Dimonte and Schneider [22, 24] and Schneider et al. [23]. The one difficult is that the present view of the RM layer does not describe the partitioning of $W_{0}$ into $h_{B 0}$ and $h_{S 0}$ which is presumably dependent on Atwood number. This partitioning for the plots was accomplished by a separate fit to the bubble and spike data based on the values of $\theta$ and $t_{0}$ and then fitting only $h_{B 0}$ and $h_{S 0}$ to the experimental data. A more exhaustive examination of experimental data sets at a variety of Atwood numbers would be necessary to determine a dependency of $h_{B 0}, h_{S 0}$ (or, more likely, the ratios) and perhaps $t_{0}$ on Atwood number. Tables I and II present the actual values determined by the fits.

The theoretically suggested range of power-law scaling exponent of the RM induced mixing layer agree reasonably well with the LEM experiments at Atwood numbers from 0.28 (density ratio about $1 / 2$ ) and 0.48 (density ratio about $1 / 3$ ). This once again supports our assertion that the establishment of the self-similar scaling for the mixing layer widths is dependent on the low wavenumber energy spectrum for a range of RM induced flows. In these Dimonte and Schneider experiments, the effects of surface tension will affect the small scales of the flow at late times. However, our present theoretical view indicates that it should not have a significant impact on the scaling of mixing layer since the scaling of both spikeside and bubble-side are assumed to depend on the large-scale property represented by the low wavenumber power-law of the energy spectrum. It must be noted that other researchers have also proposed mathematical forms that provide excellent fits to RM experiments. For example, the empirical formula provided by Shvarts et al. [3] provides excellent fits to the 
Dimonte and Schneider experiments. One objective of our efforts is to suggest a theoretical rationale for the range of observed exponents. Note also that in Zhou [4], the power-law exponent of the scaling law for the bubble side, $\theta_{B}$, of the mixing layer was evaluated. On the other hand, the analysis of the power-law exponent for the spike side was inferred from $\theta_{B}$ based on Alon et al. [21]. Our present theoretical picture gives a different, but self-consistent view of this crucial aspect of scaling analysis.

\section{DISCUSSION}

The experimental evidence cited in the previous section show that the late-time postshock evolution of the RM mixing layer is reasonable consistent with to the self-similar behavior of the decay of weakly anisotropic turbulence. The initial post-shock phase of the RM mixing layer may correspond to the early time behavior of anisotropic isotropic turbulence wherein the energy is nearly conserved, and the necessary spatial and temporal correlations are being established that will lead to the cascade of energy through an inertial range and the subsequent asymptotic decay process. Once this inertial range is established, the turbulent kinetic energy will tend toward a power-law decay in time, and the length-scale will grow as a power-law in time, as observed in experiments.

The physical picture of the RMI induced flow, and it's relationship to weakly anisotropic turbulence at the late time, was established in Zhou (2001). Zhou only considered the basic scaling for the bubbles and did not treat the scaling exponent of the spikes. Instead, the result of Alon et al. [21] was noted. In this paper, we have proposed a theoretical framework that provides the exponents for the scaling law for the spikes-it is the same exponent as for the bubbles. This analysis is a speculative extension of the theoretical and experimental turbulence work on isotropic and weakly anisotropic turbulence that has been conducted over the last fifty years. The results of this turbulence-theory-based analysis are in good agreement with the LEM experiments.

\section{CONCLUSIONS}

The analogy with weakly anisotropic decay suggests that the spike- and bubble-sides should grow as power-laws with the same power-law exponents and virtual time origins. This 
view seems to be in reasonable agreement with experiment. At very late times, the analogy with anisotropic turbulence suggests that the mixing layer will approach a "final stage of decay" wherein the turbulence interactions are dominated by viscous effects-we do not anticipate that RM experiments will achieve this regime due to the large times required (e.g., see Clark and Zemach[12]). The experiments of decaying isotropic and weakly anisotropic turbulence have not been particularly successful at providing a definitive value for the decaylaw exponent. There are a variety of reasons for this, including finite experiment size and finite Reynolds number effects (see Mohamed and LaRue[16], or Clark and Zemach[12]). One remarkable aspect of Figures 3 through 6 is the similarity between curves at late times following different power-laws.

It should be noted that the alternate view-that the two-sides of the layer decay with different power laws in time is difficult to reconcile with the usual understanding of selfsimilarity. It may be conjectured that such a state represents an intermediate asymptotic approach to partial self-similiarity that is eventually subsumed by the overall self-similarity at late times. Unfortunately the inherent physical difficulties involved in performing such experiments makes it impossible to assess if this is the case.

It should be noted that consistency with the results of the self-similar analysis is not proof that the analysis is correct. Indeed, it is expected that at high Atwood numbers the balance of physical processes and forces in the heavy fluid may be substantially different than those in the lighter fluid, and the fundamental assumption of self-similarity will be highly suspect. However, for the modest Atwood numbers tested the analysis yielded excellent agreement with experiment, and thus warrants consideration as a model of the late stages of the flow.

The empirical formula developed in [3] gives very good agreement with the LEM experiment data sets [22-24]. The results demonstrate that scaling laws can also be obtained from basic turbulence theory and are in satisfactory agreement with the LEM measurements, thus perhaps providing a more complete picture of the physical processes underlying the empirical formula in [3]. The apparent disparity of agreement at early time may indicate that the turbulence has not yet condensed to an asymptotic self-similar state.

It is perhaps unreasonable to expect that the more difficult experiments of RichtmyerMeshkov mixing layers should be any more successful at identifying power-law exponents then the simpler isotropic experiments. Nevertheless, the experiments appear to bound the power-law behaviors, and these bounds are in good agreement with the bounds presented 
here based on the large-scale (small wavenumber) scaling of isotropic turbulence.

\section{Acknowledgments}

The authors thank Dr. Guy Dimonte for providing us with his experimental data, and Dr. Mark Ulitsky, Dr. Richard Holmes and Dr. Ray Ristorcelli for discussions and helpful scrutiny of this manuscript. This work was performed under the auspices of the U.S. Department of Energy by the University of California, Lawrence Livermore National Laboratory under Contract No. W-7405-Eng-48 and Los Alamos National Laboratory under Contract No. W-7405-Eng-36. TTC also acknowledges the support by Instabilities and Turbulent Mix Project of the Nuclear Weapons Strategic Computing Program.

[1] R.D. Richtmyer, "Taylor instability in shock acceleration of compressible fluids," Comm. Pure Appl. Math, 13, 297 (2001).

[2] E.E. Meshkov, "Instability of the interface of two gases accelerated by a shock wave," Izv. Akad. Sci., USSR Fluid Dyn. 4, 101 (1969).

[3] D. Shvarts, O. Sadot, D. Oron, A. Rikanati, and U. Alon, "Shock-Induced instability of interfaces," in Handbook of Shock Waves, Volume 2: Shock Wave Interactions and Propagation, edited by G. Ben-Dor, O. Igra, and T. Elperin (Academic Press, San Diego, 2001).

[4] Y. Zhou, “A scaling analysis of turbulent flows driven by Rayleigh-Taylor and RichtmyerMeshkov instabilities," Phys. Fluids 13(2), 538 (2001).

[5] G. K. Batchelor, The Theory of Homogeneous Turbulence, (Cambridge University Press, Cambridge, 1953).

[6] P.G. Saffman, "The large-scale structure of homogeneous turbulence," J. Fluid Mech. 27(3), $581(1967)$.

[7] G. Comte-Bellot and S. Corrsin, "The use of a contraction to improve the isotropy of gridgenerated turbulence," J. Fluid Mech., 25(4), 657 (1966).

[8] T. De Kármán and L. Howarth, "On the statistical theory of isotropic turbulence," Proc. Roy. Soc. London Ser. A 164, 192 (1938).

[9] M. Lesieur, Turbulence in Fluids, (Martinus Nijhoff, Dordrecht, 1987). 
[10] M. Lesieur and D. Schertzer, “Amortissement autosimilaire d'une turbulence a grand nombre de Reynolds," J. Mec. 17, 609 (1978).

[11] T.T. Clark and C. Zemach, "A spectral model applied to homogeneous turbulence," Phys. Fluids 7(11), 1674 (1995).

[12] T.T. Clark and C. Zemach, "Symmetries and the approach to statistical equilibrium in isotropic turbulence," Phys. Fluids 10(11), 2846 (1998).

[13] T.T. Clark and Y. Zhou, "Self-similarity of two flows induced by instabilities," Submitted to Phys. Rev E, also available as Los Alamos National Laboratory Unclassified Report LAUR$02-5600$.

[14] W.C. Reynolds, "Computation of turbulent flows," Annu. Rev. Fluid Mech, 8, 183 (1976)

[15] C.G. Speziale, "Analytical methods for the development of Reynolds stress closures in turbulence," Ann. Rev. Fluid Mech, 23, 107 (1991)

[16] M.S. Mohamed and J.C. LaRue, "The decay power law in grid-turbulence," J. Fluid Mech. 219, 195 (1990).

[17] C.G. Speziale and P.S. Bernard, "The energy decay in self-preserving isotropic turbulence revisited," J. Fluid Mech. 241, 645 (1992).

[18] J.O. Hinze, Turbulence, (McGraw-Hill, New York, 1959).

[19] G.L. Eyink and D.J. Thomson, "Free decay of turbulence and breakdown of self-similarity," Phys. Fluids 12(3), 477 (2000).

[20] We are indebted to J.R. Herring for this interpretation (personal communication, Nov. 1997).

[21] U. Alon, J. Hecht, D. Ofer, and D. Shvarts, "Power laws and similarity of Rayleigh-Taylor mixing fronts at all density ratios", Phys. Rev. Lett. 74, 534 (1995).

[22] G. Dimonte and M.B. Schneider, "Turbulent Rayleigh-Taylor instability experiments with variable acceleration," Phys. Rev. E 54, 3740 (1996).

[23] M.B. Schneider, G. Dimonte, and B. Remington, "Large and small scale structure in RayleighTaylor mixing," Phys. Rev. Lett. 80, 3507 (1998).

[24] G. Dimonte and M. Schneider, "Density ratio dependence of Rayleigh-Taylor mixing for sustained and impulsive acceleration histories," Physics of Fluids, 12(2), 304 (2000).

[25] G. Dimonte, "Nonlinear evolution of the Rayleigh-Taylor and Richtmyer-Meshkov instabilities," Phys. Plasma 6, 2009 (1999).

[26] D. Oron, L. Arazi, D. Kartoon, A. Rikanati, U. Alon, and D. Shvarts, "Dimensionality de- 
pendence of the Rayleigh-Taylor and Richtmyer-Meshkov instability late-time scaling laws," Phys. Plasma 8, 2883 (2001).

[27] J.R. Ristorcelli, "Things," submitted to Phys. Fluids, 2003. 


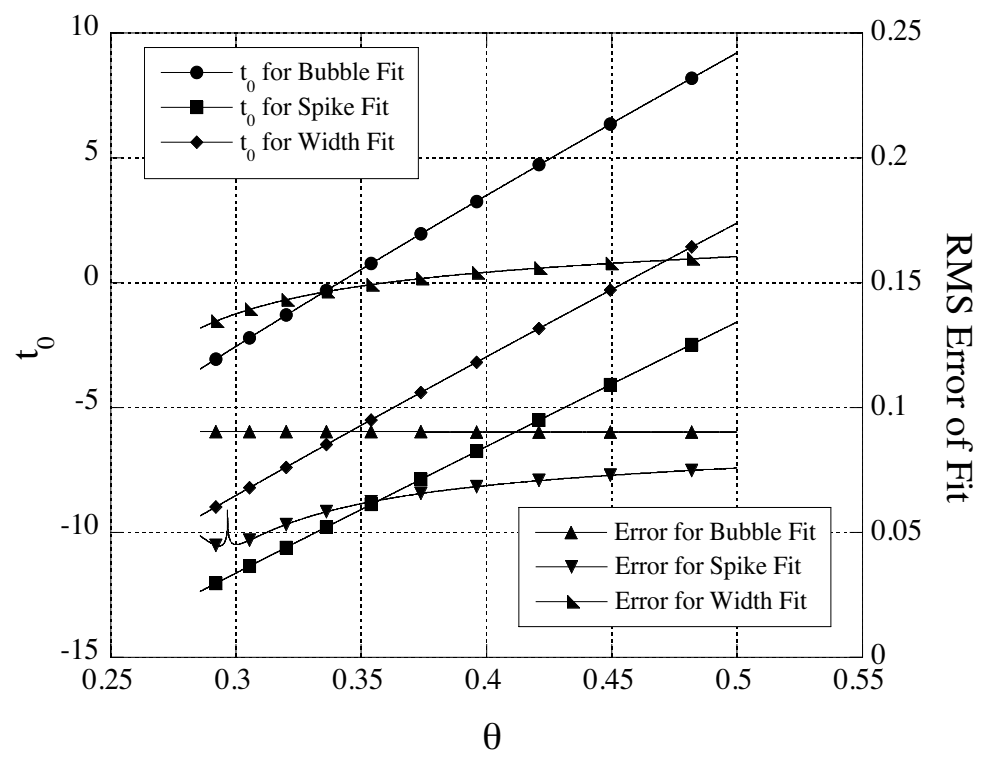

FIG. 1: Virtual time-origins and errors in fits for bubbles, spikes and widths as a function of the exponent, $\theta$, for experiments $60627-02$ and 60627-04, combined. Atwood Number $=0.22$.

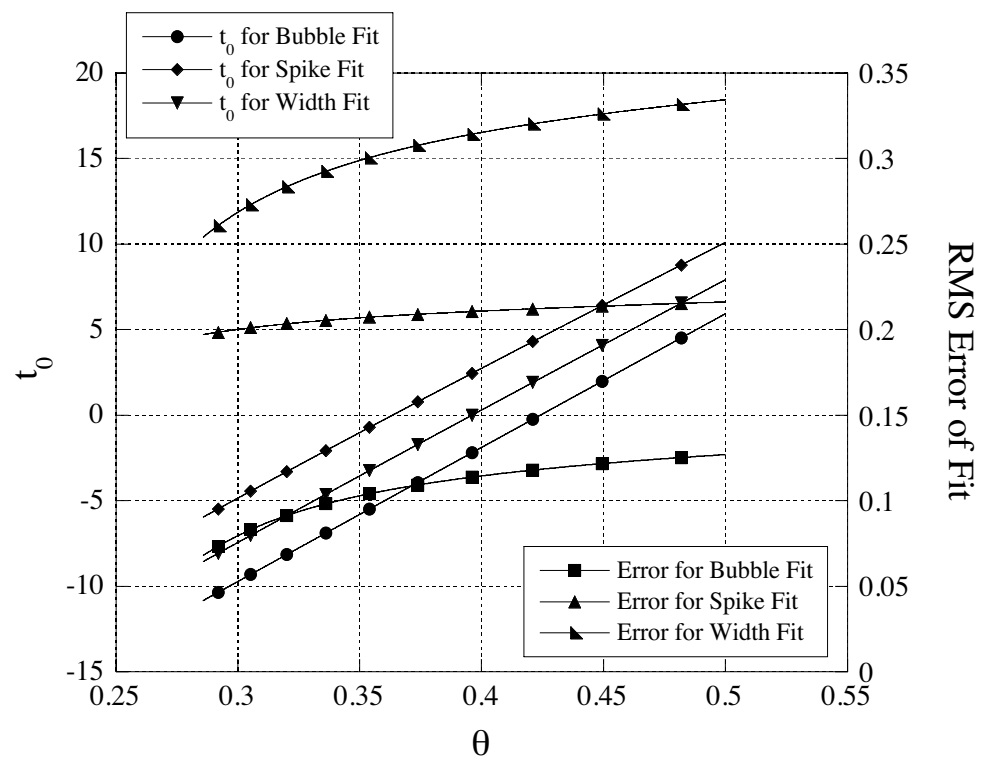

FIG. 2: Virtual time-origins and errors in fits for bubbles, spikes and widths as a function of the exponent, $\theta$, for experiments 60627-09 and 60627-10, combined. Atwood Number $=0.48$. 


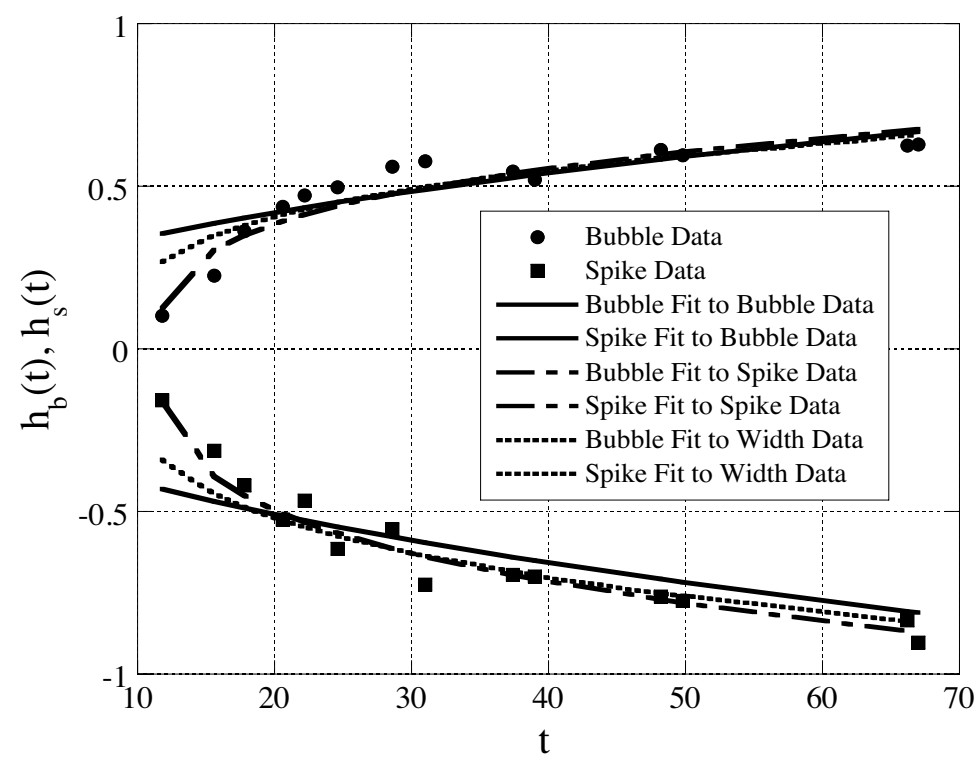

FIG. 3: Experimental data and fits for bubbles and spikes based on minimizing RMS error of fit, for experiments 60627-02 and 60627-04, combined. Atwood Number $=0.22$. Data for fits based on bubble data, $\left(\theta=0.466, t_{0}=7.278\right)$; spike data $\left(\theta=0.293, t_{0}=-11.974\right)$; and width data $\left(\theta=0.286, t_{0}=-9.340\right)$.

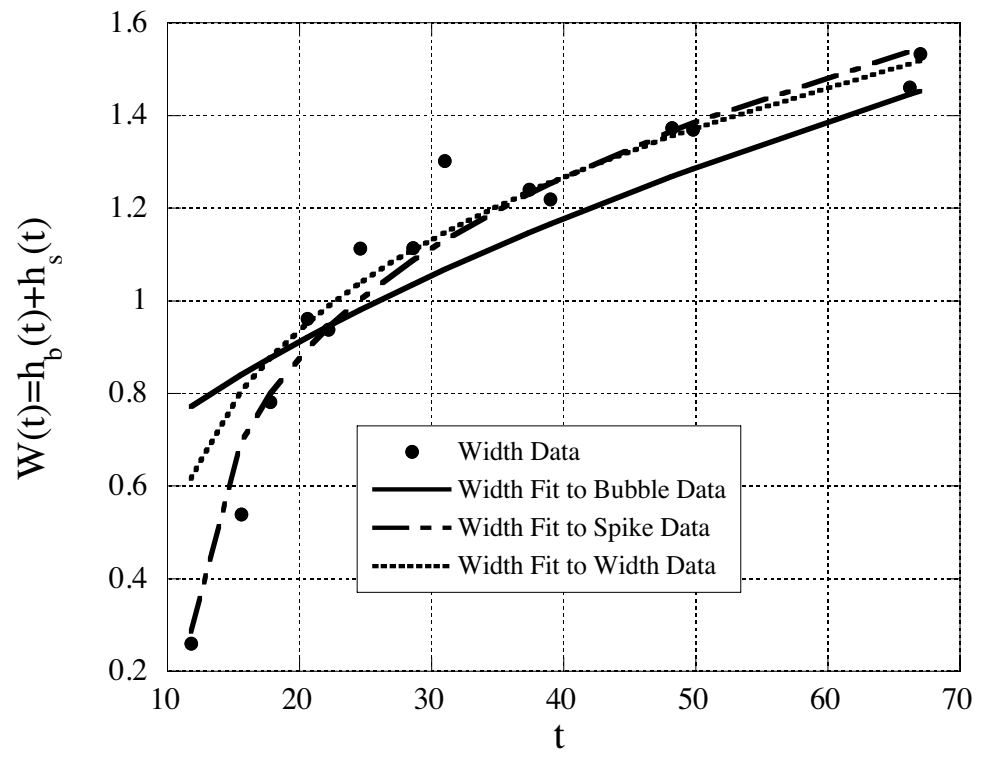

FIG. 4: Experimental data and fits for layer widths based on minimizing RMS error of fit, for experiments 60627-02 and 60627-04, combined. Atwood Number $=0.22$. Data for fits based on bubble data, $\left(\theta=0.466, t_{0}=7.278\right)$; spike data $\left(\theta=0.293, t_{0}=-11.974\right)$; and width data $\left(\theta=0.286, t_{0}=-9.340\right)$ 


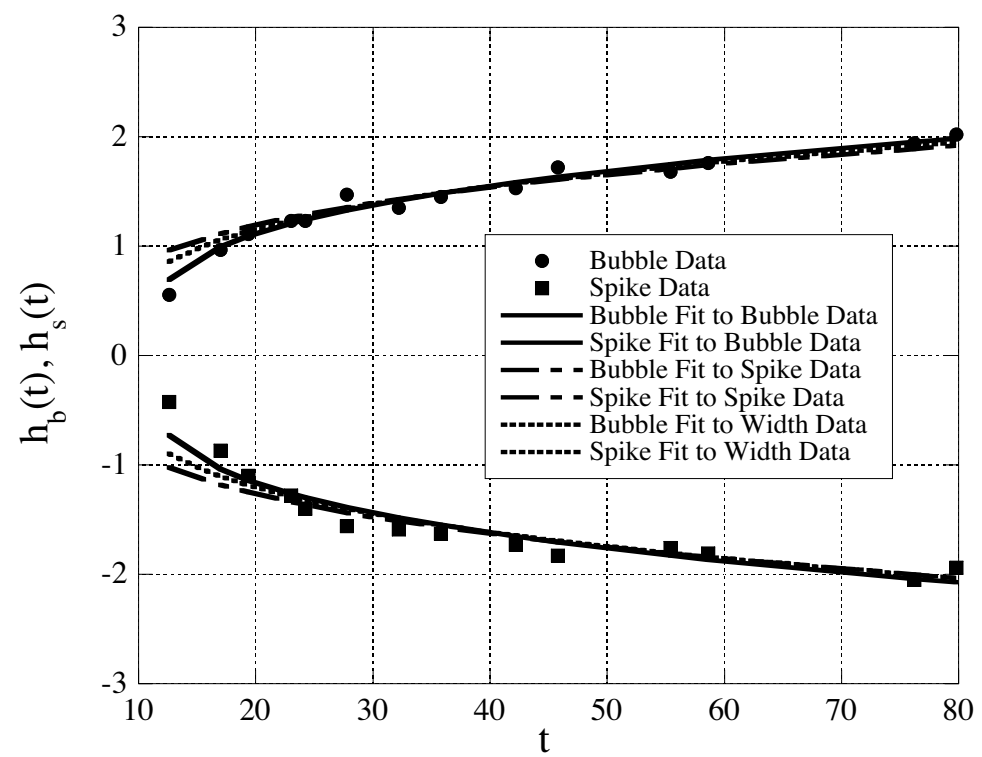

FIG. 5: Experimental data and fits for bubbles and spikes based on minimizing RMS error of fit, for experiments 60627-08 and 60627-10, combined. Atwood Number $=0.48$. Data for fits based on bubble data, $\left(\theta=0.286, t_{0}=-10.848\right)$; spike data $\left(\theta=0.286, t_{0}=-5.968\right)$; and width data $\left(\theta=0.286, t_{0}=-8.569\right)$.

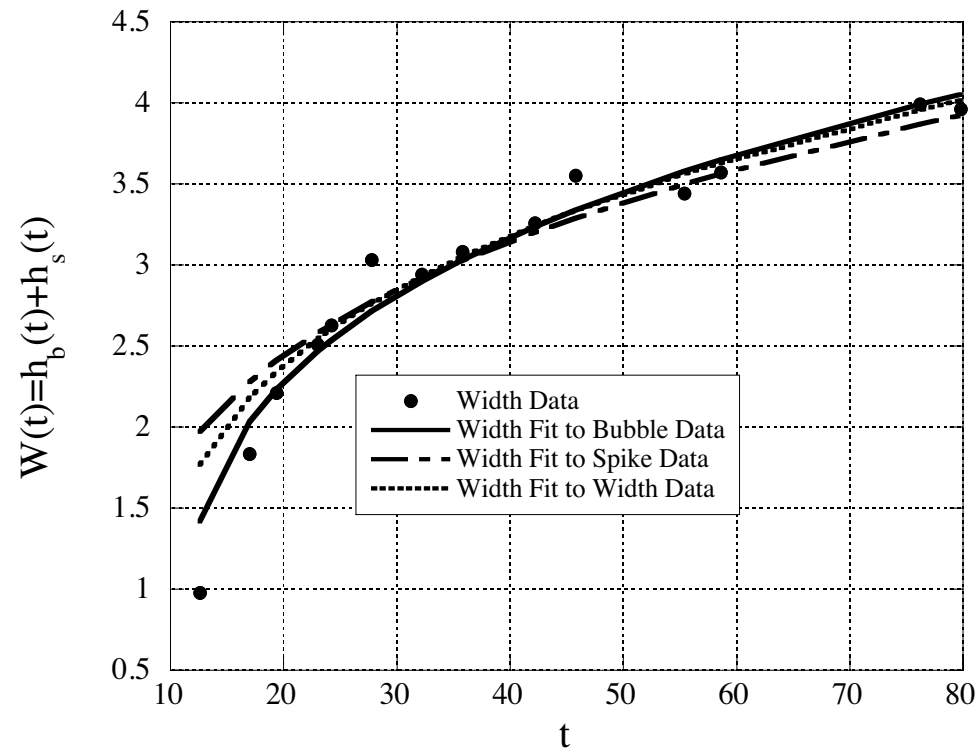

FIG. 6: Experimental data and fits for layer widths based on minimizing RMS error of fit, for experiments $60627-08$ and $60627-10$, combined. Atwood Number $=0.48$. Data for fits based on bubble data, $\left(\theta=0.286, t_{0}=-10.848\right)$; spike data $\left(\theta=0.286, t_{0}=-5.968\right)$; and width data $\left(\theta=0.286, t_{0}=-8.569\right)$. 
TABLE I: Values of $\theta$ that yield minimum errors for bubble fits, spike fits and width fits for Impulsive Rayleigh-Taylor Experiments 60627-02 and 60627-04. Data for best fits for bubble, spike and width data for each $\theta$ are included. Atwood Number $=0.22$.

$\begin{array}{lccccccc}\text { Fit } & \theta & h_{b 0} \times 10^{1} & t_{b 0} & h_{s 0} \times 10^{1} & t_{s 0} & h_{s 0} \times 10^{1} & t_{s 0} \\ \text { Bubble } & 0.4659 & 2.259 & 7.278 & 2.245 & -3.272 & 1.692 & 0.5729 \\ \text { Spike } & 0.2933 & 2.635 & -2.974 & 5.575 & -1.197 & 8.762 & -8.903 \\ \text { Width } & 0.2857 & 2.8175 & -3.457 & 5.697 & -12.37 & 9.018 & -9.3385\end{array}$

TABLE II: Values of $\theta$ that yield minimum errors for bubble fits, spike fits and width fits for Impulsive Rayleigh-Taylor Experiments 60627-08 and 60627-10. Data for best fits for bubble, spike and width data for each $\theta$ are included. Atwood Number $=0.48$.

$\begin{array}{lccccccc}\text { Fit } & \theta & h_{b 0} \times 10^{1} & t_{b 0} & h_{s 0} \times 10^{1} & t_{s 0} & h_{s 0} \times 10^{1} & t_{s 0} \\ \text { Bubble } & 0.2857 & 11.687 & -10.847 & 9.914 & -5.968 & 21.915 & -8.569 \\ \text { Spike } & 0.2857 & 11.687 & -10.847 & 9.914 & -5.968 & 21.915 & -8.569 \\ \text { Width } & 0.2857 & 11.687 & -10.847 & 9.914 & -5.968 & 21.915 & -8.569\end{array}$




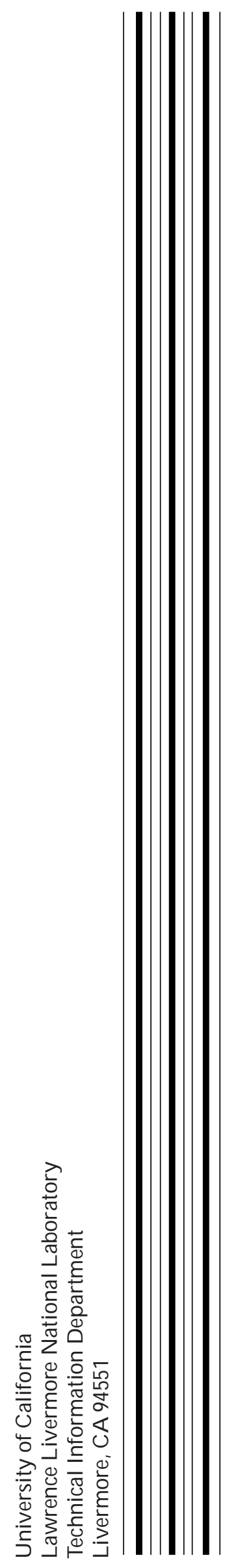

